\title{
Design of reliable silicone elastomers for dielectric elastomers and stretchable electronics
}

\author{
Mazurek, Piotr; Vudayagiri, Sindhu; Skov, Anne Ladegaard
}

\section{Published in:}

Proceedings of SPIE

Link to article, DOI:

$10.1117 / 12.2515307$

Publication date:

2019

Document Version

Publisher's PDF, also known as Version of record

Link back to DTU Orbit

Citation (APA):

Mazurek, P., Vudayagiri, S., \& Skov, A. L. (2019). Design of reliable silicone elastomers for dielectric elastomers and stretchable electronics. In Y. Bar-Cohen, \& I. A. Anderson (Eds.), Proceedings of SPIE: Electroactive Polymer Actuators and Devices (EAPAD) XXI (Vol. 10966). [109660M] SPIE - International Society for Optical Engineering. Proceedings of SPIE - The International Society for Optical Engineering https://doi.org/10.1117/12.2515307

\section{General rights}

Copyright and moral rights for the publications made accessible in the public portal are retained by the authors and/or other copyright owners and it is a condition of accessing publications that users recognise and abide by the legal requirements associated with these rights.

- Users may download and print one copy of any publication from the public portal for the purpose of private study or research.

- You may not further distribute the material or use it for any profit-making activity or commercial gain

- You may freely distribute the URL identifying the publication in the public portal 


\section{Design of reliable silicone elastomers for dielectric elastomers and stretchable electronics}

Piotr Mazurek, Sindhu Vudayagiri, Anne Ladegaard Skov

Piotr Mazurek, Sindhu Vudayagiri, Anne Ladegaard Skov, "Design of reliable silicone elastomers for dielectric elastomers and stretchable electronics," Proc. SPIE 10966, Electroactive Polymer Actuators and Devices (EAPAD) XXI, 109660M (13 March 2019); doi: 10.1117/12.2515307

Event: SPIE Smart Structures + Nondestructive Evaluation, 2019, Denver, Colorado, United States 


\title{
Design of reliable silicone elastomers for dielectric elastomers and stretchable electronics
}

\author{
Piotr Mazurek, Sindhu Vudayagiri, Prof. Anne Ladegaard Skov* \\ Technical University of Denmark, Danish Polymer Centre, Kgs. Lyngby 2800, DK \\ E-mail: al@kt.dtu.dk
}

\begin{abstract}
Silicone elastomers are widely used due to the favourable properties, such as flexibility, durable dielectric insulation, barrier properties against environmental contaminants and stress-absorbing properties over a wide range of temperatures $\approx-100{ }^{\circ} \mathrm{C}$ to $250^{\circ} \mathrm{C}$. Additionally they are mechanically reliable over millions of deformation cycles, which makes them ideal candidates for dielectric elastomers and stretchable electronics. In research on dielectric elastomers and other emerging technologies, the most common silicone elastomer utilized is Sylgard 184. One of the main advantages of this formulation is the low viscosity which allows for easy processing resulting in almost defect-free samples. Furthermore, its curing is robust and not as sensitive to poisoning as other silicone elastomer formulations. Commonly, the shortcomings of the final properties of Sylgard 184 are overcome by mixing the base polymer and the curing agent in non-stoichiometric ratios and also by blending it with softer types of commercially available elastomers. Researchers rarely formulate their own tailor-made silicone elastomers, probably due to the scarcity of information in literature on how to do this. This report aims to equip the beginners in silicone research with knowledge on how to prepare silicone elastomers with specific properties without compromising the mechanical integrity of the elastomer and thereby avoiding mechanical failure. Here the main focus is put on designing and formulating soft, reliable, and reproducible elastomers.
\end{abstract}

\section{INTRODUCTION}

Silicone elastomers are used abundantly in domestic products such as flexible baking molds, bathroom sealants, skin adhesives, cosmetics, and noise cancellation parts for car brakes, to mention but a few. Silicone elastomers are also extensively used for advanced research and technologies, in the form of stretchable electronics[1], drug delivery vehicles[2], super hydrophobic surfaces[3], and implants[4]. Silicone elastomers can be formulated to possess diverse properties that are required by the wide range of applications. Detailed manuals and informative publications about the commercially available elastomer formulations with customized additives based on their end uses (high performance, pharmaceutical, microfluidic systems, soft stretchable devices etc.,) are available from various industries and research groups.[5]-[8] However, the amount of information available in the literature on how to formulate soft and stretchable silicone elastomer networks with the good electro-mechanical stability appears insufficient. This is a crucial knowledge for furthering the dielectric elastomer technology into commercial transducers, such as actuators (electrical to mechanical energy) and generators (mechanical into electrical energy). This article will serve as a guide on how to design soft silicone elastomers that maintain good mechanical and electrical properties over repeated deformation cycles.

Commercial silicone formulations are available in various grades and many of them possess excellent mechanical properties. However, most of them possess relatively high Young's modulus which is not desirable for dielectric elastomer based actuators. Furthermore, such elastomers are not ideal for applications like stretchable electronics, where softness is a one of the key requirements. Silicone elastomers are typically crosslinked using hydrosilylation (usually Pt-catalyzed addition), condensation (usually Sn-catalyzed) or radical reactions. Based on their curing temperatures, commercial formulations are named as room temperature vulcanizing (RTV) and high temperature vulcanizing (HTV). A third type of silicone elastomer is named as liquid silicone rubber (LSR/LR), which is usually cured at high temperatures but with a distinction from HTV being that it is formulated to possess lower viscosity. A commercially available silicone elastomer kit of an addition curing system consists of two premixes named ' $A$ ' and ' $B$ ', where most commonly one of them is denoted

Electroactive Polymer Actuators and Devices (EAPAD) XXI, edited by Yoseph Bar-Cohen, lain A. Anderson, Proc. of SPIE Vol. 10966, 109660M - (C) 2019 SPIE · CCC code: 0277-786X/19/\$18 · doi: 10.1117/12.2515307 
as a crosslinker and the other a base or a polymer. Usually, the base or polymer premix contains the polymer and a catalyst. The crosslinker premix contains a crosslinker and some functional polymer or potentially silicone oil. Usually, silica particles or resins are added to this premix as well. Resins need to be added in very high concentrations in order to give mechanical properties comparable to the silica filled elastomers. However, the resulting elastomers have better transparency. The silica particles are usually fumed but may also be chemically surface-treated[9] for better compatibility with the silicone polymers. Silicone elastomers with no silica or resins have poor mechanical properties and are not recommended for applications where good mechanical stability is desired.

It is always advisable to prepare commercial formulations according to mixing ratios supplied by the manufacturer in order to obtain reliable networks. Changing the mixing ratio of part A and B to make softer elastomers may lead to loss of mechanical integrity since it causes imperfect network structures. For an excess of crosslinker, the resulting elastomer contains an excess of Si-H groups, which are prone to hydrolyze and further crosslink. Excess of Si-vinyl gives complex time-dependent viscoelasticity and thus the mechanical properties of the elastomer become strongly dependent on the applied strain rate.

\section{NETWORK STRUCTURE}

In order to understand elastomers' properties and to design them for specific uses, it is important to understand how the choice of reactants and curing parameters affect the final properties. First, we will focus on the curing chemistries and subsequently the concept of an ideal network and an imperfect network is discussed.

\subsection{Ideal networks}

An ideal elastomer network is formed by irreversible covalent bonding of a polymer and crosslinker such that elastic behavior is not transient in the presence of large strains or increased temperatures. When the connections are covalent in nature, the elastomer is denoted as a 'thermoset'. For most of the thermosets, curing is an irreversibly process and resulting elastomers cannot be reheated to allow for remolding. Thermosets are, however, usually used to describe hard and rigid materials, and the term elastomer is used to distinguish soft and elastic thermosets from the rigid counterparts. Recently, novel chemistries have allowed for preparation of thermoplastic silicone elastomers, in which the connections are transient. The thermoplastic elastomers allow for switch on-off elasticity and can be recycled.[10] However, in this instance, temperature robustness is compromised due to melting at elevated temperatures.

Theoretically, the simplest possible polymer network is prepared from the reaction of a crosslinker with more than two reactive groups per molecule (functionality $f>2$ ) and a polymer with a functionality of 2 . The stoichiometry, $r$, of the reaction, which is a ratio between the two types of reactive groups, is defined as:

$$
r=\frac{[\text { hydride }]}{[\text { vinyl }]}=\frac{f[\text { crosslinker }]}{2[\text { polymer }]}
$$

In the above equation (1), the square brackets indicate molar concentrations. When the reaction between the crosslinker and the polymer is equimolar (i.e. equal concentrations of hydride and vinyl groups), the reactions are called stoichiometric and $r=1$. Often, stoichiometric conditions are targeted. However, as mentioned earlier, changing the stoichiometry is considered as an efficient method that allows for tuning elastic properties, since stoichiometry governs the network structure. This will be discussed later.

Furthermore, in the simplest case, intra-reactions do not take place, and the network formation is based solely on the reaction between the functional groups of the crosslinker and the polymer. Side reactions are assumed to be non-existent. This is usually denoted as a 'model system', since network imperfections, such as elastically inactive loops, can be ignored under such ideal conditions. The system of telechelic vinyl-functionalized linear PDMS and an oligomeric (i.e. short) multifunctional hydride-functional siloxane crosslinker is regarded as a model system and is thus ideal for studying the dynamics of polymer networks.[11] The reaction of a three-functional crosslinker (3f) is illustrated in Figure 1.

The involved crosslinking reaction, commonly referred to as 'hydrosilylation', between the hydride and the vinyl groups proceeds almost to completion, i.e. high conversion. Side reactions are limited, i.e. the reaction is very selective.[11]-[13] 
Thereby, the final network consists of elastically active polymer chains connected at both ends to a crosslinker, as illustrated in Figure 1. The elastic modulus, E, of this ideal network (also commonly denoted Young's modulus), with elastic chains acting as entropic springs, can then be calculated as:

$$
E=v_{c} R T=\frac{f \rho_{\text {polymer }}}{2 M_{\text {polymer }}} R
$$

In equation (2), $v_{c}$ is crosslinking density, $R$ is the gas constant and $T$ is the absolute temperature. $\rho_{\text {polymer }}$ is the density of the polymer (usually around $0.98 \mathrm{~g} / \mathrm{cm}^{3}$ for polydimethylsiloxane (PDMS) based elastomers), while $M_{\text {polymer }}$ is the number average molecular weight of the polymer (abbreviated in the following as $M$ ). The equation (2) holds only for stoichiometric networks in which a low molecular weight crosslinker was used and with no fillers were added. The elastic modulus is also the commonly denoted as Young's modulus, which is symbolized with either $E$ or $Y$.

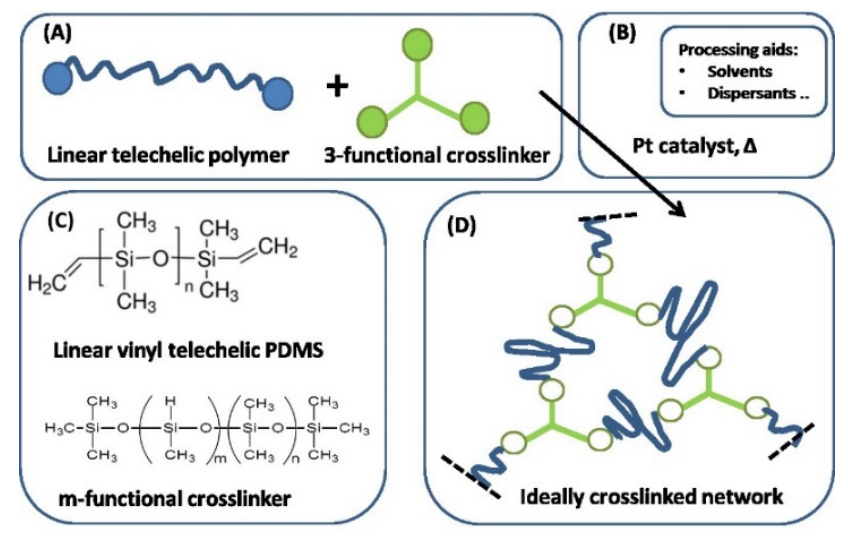

Figure 1. Illustration of a pathway to an ideal network by using a three-functional crosslinker. (A) Reactants. (B) Catalyst. (C) Structure of the utilized polymers. (D) The resulting ideal network consists of one single molecule.[14]

\subsection{Imperfect networks}

The network illustrated in Figure 1D is ideal for visualization and this is by far not the true structure of real networks, which consist of unreacted functional groups due to steric hindrance or as a result of side reactions diminishing the targeted stoichiometric balance. Side reactions and/or stoichiometrically imbalanced reactants (i.e. $r \neq 1$ ) result in dangling polymer chains and substructures as well as a sol fraction, i.e. a fraction not covalently connected to the polymer network.[15] If the reactants are mixed in an off-stoichiometric ratio, a true network is not formed. This off-stoichiometric reaction leads, in extreme cases, to a viscoelastic liquid composition consisting of highly branched polymers, and this implies that the gelation threshold has not been exceeded. The gelation threshold (also often denoted as gel point) can be defined as a lower (too much polymer) and higher boundary (too much crosslinker) of $r$ according to the Flory-Stockmayer theory:[16],[17]

$$
\begin{aligned}
& r_{c, \text { lower }}=\frac{1}{f-1} \\
& r_{c, \text { upper }}=f-1
\end{aligned}
$$

This theory is based on ideal conditions with full conversion and no imperfections, but it provides a good estimate of critical stoichiometry. For the formulation of silicone-based adhesives, the lower bound is commonly targeted to yield structures with a large fraction of dangling structures that provide excellent adhesion to, for example, human skin.[18] At $r=1$ there are elastically active chains only (ideally). The two gelation points (as defined in equations (3) and (4)) are reached when the volume fractions of the elastically active network reach 0.[14] 
An illustration of so-called 'first order' imperfections (dangling chains) is given in Figure 2. Higher order dangling structures (i.e. with multiple sets of constituents in each highly branched structure) become structurally very complex and contribute with very slow dynamics.[19] The most complex of these are encountered close to the two gel points, as defined above, and the most common examples are illustrated in Figure 3. The sol fraction gives rise mainly to viscous behavior, but in an entangled state it may cause time-dependent elasticity, which results from the polymer chains not being able to release stress immediately during fast deformation, in which case they act elastically until all stress is released.[20]

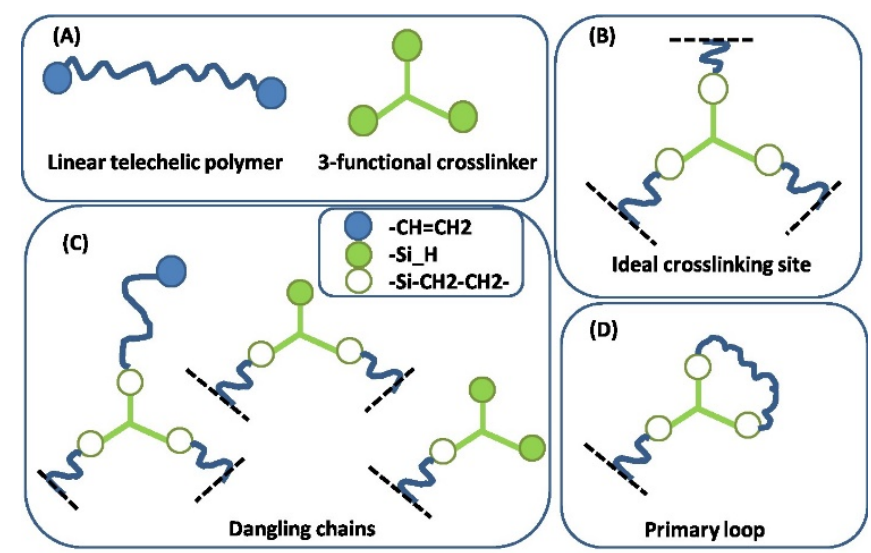

Figure 2. Some common first order imperfections in networks. (A) The two constituents in the network (reactants). (B) An ideal, elastic junction. The black dashed lines indicate connections to the infinite network. (C) Dangling chains of first order, i.e. dangling structures, which consist solely of one of each constituent or a set thereof. (D) A primary inelastic loop.[14]

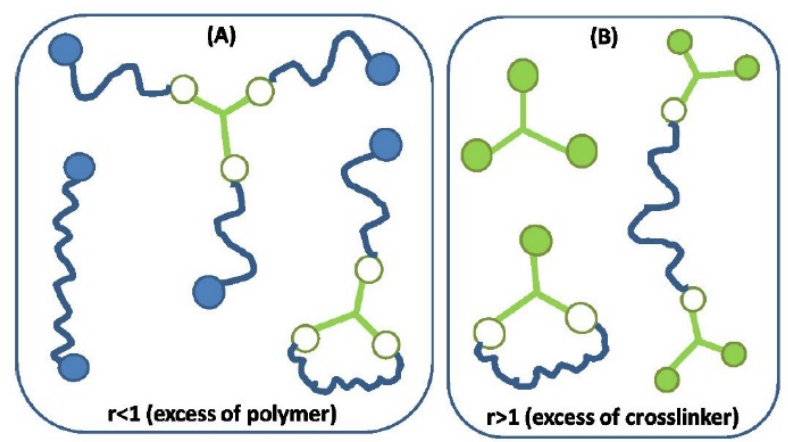

Figure 3. Illustration of the most common sol structures for the two scenarios of (A) excess of polymer and (B) excess crosslinker. Networks resulting from $\mathrm{r}<1$ possess by far the most complex dynamical behavior, whereas those resulting from $\mathrm{r}>1$ may be prone to curing over time when the utilized crosslinker is hydride-functional.[14]

Furthermore, most commercial formulations contain a fraction of non-functional or monofunctional polymers (telechelic polymers cannot be prepared with 100\% yield) that will also increase the amount of imperfections in the network. Finally, loop formation, i.e. polymers reacting at both ends with the same crosslinker, may occur and lead to loss of the effective number of elastic strands. All of these network imperfections decrease the effective crosslinking density (and thus also the elastic modulus) and introduce strongly time-dependent (transient) elasticity if the deformation is faster than the given relaxation time of the dangling substructure. Fast deformation results in the substructures acting elastically during the process but relax thereafter implying losses. 


\section{FORMULATION OF SILICONE ELASTOMERS}

In this section, different recommendations and considerations for the design of the network structure are provided, to allow the reader to develop a qualified starting point for formulating the desired silicone elastomer.

\subsection{Designing the network structure}

The network structure determines elasticity to a large extent, and therefore various variables for network preparation will be discussed. The first part of the formulation process is to decide which elastic modulus is being targeted. It is complicated to formulate reliable silicone elastomers with elastic moduli below 0.6 MPa, since this is the apparent elastic modulus of well-entangled silicone polymers (i.e. silicone polymers above approximately 12,000 g/mol). The entanglements in crosslinked polymers behave as crosslinks with more dynamic behavior, since they can slide in between each other and some of them can also disentangle. Those that can disentangle are commonly denoted as 'trapped', whereas ones that cannot do so are denoted as 'locked'. They give rise to a constant contribution to elasticity when the polymer length is above the entanglement molecular weight. Above this length, the concentration of entanglement remains constant and thus a constant contribution to the modulus. Recently, so-called 'bottlebrush' elastomers have been developed and they bypass this limitation, due to effectively changing the molecular entanglement weight.[21],[22] More details on entanglements and the characteristic properties of silicone polymers can be found in the article by Fetters et al.[23] Alternatively, elastomers can be processed in the presence of a large amount of solvent, in order to dilute locked entanglements[24] and thereby proceed below 0.6 MPa. However, this process is not always favorable, since the solvent may swell other components in the final devices.

Silicone elastomers with elastic moduli above $1 \mathrm{MPa}$ : Traditionally, for formulating high-modulus elastomers, the stoichiometry $r=1.1-1.4$, the crosslinker functionality $f \geq 8$, and the polymer chain length before crosslinking $M \approx 20,000$ to $70,000 \mathrm{~g} / \mathrm{mol}$ can be used. Silica fillers can be introduced in significant amounts, and so relatively high tear strength and electrical breakdown strength can also be achieved. For low viscosity premixes, $M<20,000 \mathrm{~g} / \mathrm{mol}$ can be used. Too low molecular weight causes the elastomers to become brittle.

Silicone elastomers with elastic moduli below $1 \mathrm{MPa}$ : For soft elastomers the stoichiometry $r<1$, the crosslinker functionality $\mathrm{f} \geq 8$ and the molecular weight of the polymer chains $M \approx 50,000 \mathrm{~g} / \mathrm{mol}$ can be used. If tear strength is not a criterium then silica particles can be fully or partly omitted making easier to achieve lower elastic modulus. If tear strength is important, a low Young's modulus is difficult to achieve but it can be achieved by simultaneous entanglement dilution, reduction of filler content and decreased stoichiometry. For formulating much softer silicones to be employed as adhesives, the stoichiometry should be lower than 1 and higher than the $\sim r_{c, \text { lower }}$, and $M \approx 70,000 \mathrm{~g} / \mathrm{mol}$ and upwards. Polydisperse polymers are also favorable for adhesives.

Silicone elastomers with high extensibility: Highly extensible formulations generally should have a stoichiometry $r<1$, crosslinker functionality $f=4-5$, and molecular weight of polymer chain $M \approx 70,000 \mathrm{~g} / \mathrm{mol}$ and upwards. For moderately extensible elastomers, $r \approx 1, \mathrm{f} \geq 8$ and $M \approx 50,000 \mathrm{~g} / \mathrm{mol}$ and upwards should be employed. A novel, very high-elongation (up to $5000 \%$ ) elastomer system has been developed by Goff et al.[25] and commercialized by Gelest as ExSil 100. The underlying principle here is the reaction of a silicone polymer with hydride-functionality at one end and vinyl at the other end. In this instance, in theory, only one big molecule may result from the inherent stoichiometric reaction.

\section{MECHANICAL PROPERTIES OF SILICONE ELASTOMERS}

Understanding the mechanical properties of silicone elastomers is essential to direct their usage and to properly report mechanical data for the research community. Testing soft elastomers is usually more complex compared to hard elastomers. Therefore, it is always crucial to use a tensile testing machine with the right sensitivity for the materials under investigation. It is important to evaluate mechanical data critically, as a common fault in the literature is to report the ultimate properties 
with multiple significant numbers relying on one single measurement or without providing standard deviations. Ultimate properties are as such not intrinsic properties since imperfections within the elastomer govern the resulting properties.

In the case of ideal, soft elastomers, there are three primary mechanical properties of interest, namely 1) elastic modulus, 2) either ultimate stress or strain and 3) tear strength. For non-ideal elastomers the relationship between stress and strain becomes non-linear and is dependent on the deformation rate.

\subsection{Elastic modulus}

Silicone elastomers are viscoelastic i.e. they possess both elasticity and viscous dissipation, with the main contribution being elasticity. In terms of mechanical properties, their elastic moduli are usually very low (of the order of a few MPa as a maximum) and they are very extensible, with ultimate strains usually far above $300 \%$.

The ideal polymeric network is typically illustrated by means of a grid, to show the three-dimensional structure of the network with all reactive groups participating in covalent crosslinks and yielding ideal inter-connections between crosslinkers. The crosslinking points between the polymer chains are illustrated as elastic strands, visualized as springs connected via the crosslinker. The spring changes its entropy and is thus denoted as an entropic spring. The spring constant of the series of interconnected entropic springs is then the elastic modulus, and thus it is proportional to crosslinking density. This is the basis for the derivation for Hooke's law.

From the assumption of incompressibility (Poisson's ratio $v=1 / 2$ ) of the elastomer, following relation coupling, the elastic modulus $(E)$ and the shear modulus $(G)$ is derived:

$$
E=3 G
$$

Hooke's law is valid only for small strains, usually up to $10-15 \%$ for silicone elastomers (but can be significantly larger e.g. $30 \%$ ) with limited amounts of filler. Beyond the linear regime, the elastomers may strain-harden (the slope of the stress-strain plot increases) or strain-soften (the slope decreases).[26] Strain-softening of soft silicone elastomers at strains above approximately $50 \%$ is usually a sign of poor network structure. Reliable silicone elastomers must have either an almost constant slope or contain a small increase at higher strains. Filled, harder silicone elastomers may show natural stress-softening at larger strains, due to disappearance of particle-particle interactions. This usually causes some extent of plasticity, i.e. lack of reversibility.

\subsection{Ultimate properties}

Ultimate properties are the maximum strain and maximum stress before break. In general, a softer silicone elastomer results in larger ultimate strain and lower ultimate stress. Unlike the elastic moduli, the ultimate stress and strain are not entirely material parameters as they rely strongly on the number of imperfections in the network. A poorly cured elastomer may result in a significantly higher maximum strain and lower maximum stress than the ideally cured version, while a poorly processed silicone elastomer with air bubbles introduced in the coating process, may result in both lower ultimate stress and strain. Therefore, variations in ultimate property values are expected as presented in Figure 4 where mechanical properties of an elastomer based on very high-viscosity glycerol-silicone formulation is shown. Hence, the ultimate properties should be assessed based on at least three to five measurements, to provide any valuable information. 


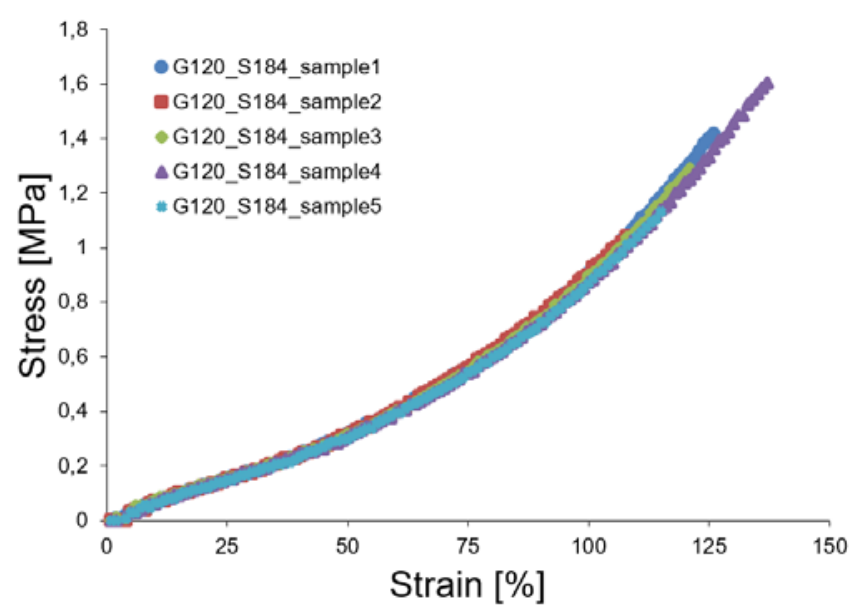

Figure 4. Stress-strain curves of a glycerol-silicone elastomer formulation.[27],[28] These 5 specimens show extensions between 115 $\%$ and $135 \%$ with the ultimate stress ranging from 1.1 to $1.6 \mathrm{MPa}$.

\subsection{Non-linear mechanical properties of filled elastomers}

For very soft elastomers, shear experiments are easier to perform than extensional experiments, and it is therefore convenient to determine the elastic modulus from oscillatory shear with a given angular frequency $(\omega)$. This method, though, must be used with precaution when dealing with filled elastomers. From shear experiments, one obtains the elastic contribution from the material (opposing force), which is denoted as the storage modulus, $G^{\prime}(\omega)$ as well as the loss modulus, $G^{\prime \prime}(\omega)$ which is the energy dissipated. However, the correlation in the case of filled elastomers should be specified strictly to:

$$
E=3 G_{0}^{\prime}
$$

where $G_{0}^{\prime}=G^{\prime}(\omega \rightarrow 0)$. The need for this definition is not obvious for silicone elastomers with a small amount of particles only, since the storage modulus is almost independent of the applied angular frequency. The particles contribute with their own dynamics, and they do this in complex ways, simply due to an effect of the particles on the elastic modulus. Three complex and highly non-linear phenomena are often encountered for filled silicone elastomers:

1. Mullins effect: the filled elastomers 'remember' the maximum extension that they have experienced. The higher the maximum extension a sample has experienced, the lower the elasticity.

2. Payne effect: the filled elastomer has two plateaus in the shear moduli. At small deformation rates, the particles act in an elastic manner and contribute significantly to elasticity.

3. Permanent set: the filled elastomer does not return to the original zero-stress at no strain after it has been held at a given strain for an extended period of time. Rather, it extends a few per cent due to reorganizing the elastomer and potentially also post-curing in the extended state. This is also denoted plasticity.

More information on these phenomena can be found in reference [14] and the references within.

\subsection{Practical precautions with respect to the three non-linear phenomena}

For reproducibility and reliability, the elastomer should always be stretched significantly beyond what it has experienced during processing, thereby ensuring that the elastomer films have experienced the same maximum strain. In practice, elastomers with strong Payne effects in dynamic applications operated at high speeds may possess a certain strain rate at which the elastomer softens strongly. For determining the elastic properties of elastomers, it is important to ensure that the obtained linear viscoelastic (LVE) diagram corresponds to the utilized frequency in the application. The permanent set must be evaluated and be considered in design of products. Since permanent set strongly increases with particle loading, low filler concentrations should be used if possible. More detailed explanations of the three phenomena can be found in the references [29]-[31] and references therein. 


\section{SUMMARY AND CONCLUSIONS}

Formulation strategies are not easily accessible for academia and knowledge about elastomer formulating and optimization for e.g. soft electronics and dielectric elastomers remains relatively limited. With many new silicone-based technologies emerging commercial elastomers are experiencing a bit of a shortfall since they are usually formulated with focus on relatively hard and durable elastomers. It is essential and advantageous for researchers and product developers to formulate their own silicone elastomer recipes for improved product performance in order not to be constrained by commercial formulations. The correct formulation of silicone elastomers allows for a wide range of mechanical properties while maintaining mechanical integrity. The formulation strategy explained here is only a starting point and can be expanded easily to incorporate silicone copolymers of various types and to include additives and fillers. The properties of silicone elastomers can be varied drastically by designing the network and additives carefully. The number of choices for elastomer reactants and additives is very high and allows for a wide range of unexplored possibilities for self-formulated silicone elastomers.

\section{Conflicts of interest}

There are no conflicts to declare.

\section{Acknowledgements}

Authors have reproduced some material from [14] (https://pubs.rsc.org/en/content/articlehtml/2019/cs/c8cs00963e) with permission from the Royal Society of Chemistry.

The authors acknowledge funding from the Independent Research Council Denmark.

\section{REFERENCES}

[1] M. G. Mohammed and R. Kramer, “All-Printed Flexible and Stretchable Electronics,” Adv. Mater., vol. 29, p. 1604965, 2017.

[2] P. Mazurek, M. A. Brook, and A. L. Skov, “Glycerol-Silicone Elastomers as Active Matrices with Controllable Release Profiles,” Langmuir, vol. 34, pp. 11559-11566, 2018.

[3] H. Zhou, H. Wang, H. Niu, A. Gestos, X. Wang, and T. Lin, "Fluoroalkyl silane modified silicone rubber/nanoparticle composite: A super durable, robust superhydrophobic fabric coating,” Adv. Mater., vol. 24, no. 18, pp. 2409-2412, 2012.

[4] C. T. K. Khoo, "Silicone synovitis: The current role of silicone elastomer implants in joint reconstruction,” J. Hand Surg. (British Eur. Vol., vol. 18, no. 6, pp. 679-686, 1993.

[5] A. Tiwari and M. D. Soucek, Eds., Concise Encyclopedia of High Performance Silicones. Scrivener Publishing, 2014.

[6] K. Mojsiewicz-Pieńkowska, "Review of Current Pharmaceutical Applications of Polysiloxanes (Silicones)," Handb. Polym. Pharm. Technol., vol. 2, no. August 2015, pp. 363-381, 2015.

[7] M. P. Wolf, G. B. Salieb-Beugelaar, and P. Hunziker, "PDMS with designer functionalities—Properties, modifications strategies, and applications,” Prog. Polym. Sci., vol. 83, pp. 97-134, 2018.

[8] S. Park, K. Mondal, R. M. Treadway, V. Kumar, S. Ma, J. D. Holbery, and M. D. Dickey, “Silicones for Stretchable and Durable Soft Devices: Beyond Sylgard-184,” ACS Appl. Mater. Interfaces, vol. 10, no. 13, pp. 11261-11268, 2018.

[9] S. Rezaei and B. Pourabbas, "In situ generation of the surface-modified silica nanoparticles within the silicone matrix," Polym. Compos., vol. 36, no. 7, pp. 1365-1370, 2015.

[10] A. S. Fawcett and M. A. Brook, "Thermoplastic silicone elastomers through self-association of pendant coumarin groups," Macromolecules, vol. 47, no. 5, pp. 1656-1663, 2014.

[11] H. H. Winter and F. Chambon, “Analysis of Linear Viscoelasticity of a Crosslinking Polymer at the Gel Point,” J. Rheol. (N. Y. N. Y)., vol. 30, no. 2, pp. 367-382, 1986. 
[12] M. A. Villar, M. A. Bibbo, and E. M. Valles, "Influence of Pendant Chains on Mechanical Properties of Model Poly(dimethylsiloxane) Networks. 1. Analysis of the Molecular Structure of the Network,” Macromolecules, vol. 29, no. 11, pp. 4072-4080, 1996.

[13] M. A. Villar and E. M. Vallés, "Influence of pendant chains on mechanical properties of model poly(dimethylsiloxane) networks. 2. Viscoelastic properties,” Macromolecules, vol. 29, no. 11, pp. 4081-4089, 1996.

[14] P. Mazurek, S. Vudayagiri, and A. L. Skov, "How to tailor flexible silicone elastomers with mechanical integrity: a tutorial review,” R. Soc. Chem., pp. 1-18, 2019.

[15] S. M. G. Frankær, M. K. Jensen, A. G. Bejenariu, and A. L. Skov, "Investigation of the properties of fully reacted unstoichiometric polydimethylsiloxane networks and their extracted network fractions,” Rheol. Acta, vol. 51, no. 6, pp. 559567, 2012.

[16] P. J. Flory, “Molecular Size Distribution in Three Dimensional Polymers. I. Gelation,” J. Am. Chem. Soc., vol. 63, no. 11, pp. 3083-3090, 1941.

[17] W. H. Stockmayer, “Theory of Molecular Size Distribution and Gel Formation in Branched Polymers II. General Cross Linking,” J. Chem. Phys., vol. 12, no. 4, pp. 125-131, 1944.

[18] S. Sun, M. Li, and A. Liu, “A review on mechanical properties of pressure sensitive adhesives,” Int. J. Adhes. Adhes., vol. 41, pp. 98-106, 2013.

[19] A. L. Larsen, K. Hansen, P. Sommer-Larsen, O. Hassager, A. Bach, S. Ndoni, and M. Jørgensen, "Elastic properties of nonstoichiometric reacted PDMS networks,” Macromolecules, vol. 36, no. 26, pp. 10063-10070, 2003.

[20] A. L. Skov and L. Yu, “Optimization Techniques for Improving the Performance of Silicone-Based Dielectric Elastomers,” Adv. Eng. Mater., vol. 20, p. 1700762, 2017.

[21] J. Paturej, S. S. Sheiko, S. Panyukov, and M. Rubinstein, "Molecular structure of Bottlebrush polymers in melts,” Sci. Adv., vol. 2, no. 11, p. 1601478, 2016.

[22] L. H. Cai, T. E. Kodger, R. E. Guerra, A. F. Pegoraro, M. Rubinstein, and D. A. Weitz, "Soft Poly(dimethylsiloxane) Elastomers from Architecture-Driven Entanglement Free Design,” Adv. Mater., vol. 27, no. 35, pp. 5132-5140, 2015.

[23] L. J. Fetters, D. J. Lohse, D. Richter, T. A. Witten, and A. Zirkel, “Connection between Polymer Molecular Weight, Density, Chain Dimensions, and Melt Viscoelastic Properties,” Macromolecules, vol. 27, no. 17, pp. 4639-4647, 1994.

[24] A. L. Larsen, P. Sommer-Larsen, and O. Hassager, "Dynamic dilution effects in polymeric networks,” E-Polymers, vol. 42, pp. 1-11, 2006.

[25] J. Goff, S. Sulaiman, B. Arkles, and J. P. Lewicki, “Soft Materials with Recoverable Shape Factors from Extreme Distortion States,” Adv. Mater., vol. 28, no. 12, pp. 2393-2398, 2016.

[26] T. K. Kim, J. K. Kim, and O. C. Jeong, "Measurement of nonlinear mechanical properties of PDMS elastomer," Microelectron. Eng., vol. 88, no. 8, pp. 1982-1985, 2011.

[27] P. Mazurek, L. Yu, R. Gerhard, W. Wirges, and A. L. Skov, "Glycerol as high-permittivity liquid filler in dielectric silicone elastomers,” J. Appl. Polym. Sci., vol. 133, pp. 1-8, 2016.

[28] P. Mazurek, S. Hvilsted, and A. L. Skov, "Green silicone elastomer obtained from a counterintuitively stable mixture of glycerol and PDMS,” Polymer (Guildf)., vol. 87, pp. 1-7, 2016.

[29] L. Mullins, “Softening of Rubber by Deformation,” Rubber Chem. Technol., vol. 42, no. 1, pp. 339-362, 1969.

[30] A. R. Payne, “The dynamic properties of carbon black-loaded natural rubber vulcanizates. Part I,” J. Appl. Polym. Sci., vol. 6, no. 19, pp. 57-63, 1962.

[31] L. R. G. Treloar, “Crystallisation phenomena in raw rubber,” Trans. Faraday Soc., vol. 37, pp. 84-97, 1941. 